\title{
Advanced Fusion Reactors for Space Propulsion and Power Systems
}

\author{
John J. Chapman, NASA, Langley Research Center
}

In recent years the methodology proposed for conversion of light elements into energy via fusion has made steady progress. Scientific studies and engineering efforts in advanced fusion systems designs have introduced some new concepts with unique aspects including consideration of Aneutronic fuels. The plant parameters for harnessing aneutronic fusion appear more exigent than those required for the conventional fusion fuel cycle. However aneutronic fusion propulsion plants for Space deployment will ultimately offer the possibility of enhanced performance from nuclear gain as compared to existing ionic engines as well as providing a clean solution to Planetary Protection considerations and requirements. Proton triggered ${ }^{11}$ Boron fuel $\left(p-{ }^{11} \mathrm{~B}\right)$ will produce abundant ion kinetic energy for In-Space vectored thrust. Thus energetic alpha particles "exhaust " momentum can be used directly to produce high ISP thrust and also offer possibility of power conversion into electricity. $p-{ }^{11} \mathrm{~B}$ is an advanced fusion plant fuel with well understood reaction kinematics but will require some new conceptual thinking as to the most effective implementation.

\section{Traditional Fusion reaction: $\mathrm{D}+\mathrm{T} \rightarrow \mathrm{n}(14.07 \mathrm{MeV})+{ }^{4} \mathrm{He}(3.52 \mathrm{MeV})$}

The deuterium-tritium fueled plasma has been widely envisioned for terrestrial power plant reactors. D-T has been calculated to provide an ultimate theoretical nuclear gain ranging from $\sim 450$ to 1000 . The $\mathrm{D}$-T reaction liberates $\sim 80 \%$ of its energy via the release of $14 \mathrm{MeV}$ neutrons. The overall reaction produces additional neutrons from reactions with tritium. Additional neutrons may be released as by-products of branch reactions. The D-T reaction consumes approx. $226 \mathrm{Kg}$ of Tritium per GW of energy produced. Although energetically productive, the $\mathrm{D}-\mathrm{T}$ reaction produces potentially harmful radiation products that render this approach less attractive as regards radiation protection on-board a spacecraft or with respect to ascent or re-entry phase where the public safety is at risk. Requirements related to radiation shielding and capture blankets preclude a lower overall system mass.

An abundant alternative clean fusion fuel approach uses $\mathrm{p}^{11}$ Boron fuel.

Advanced aneutronic nuclear fusion fuels include:

$$
\begin{aligned}
& { }^{3} \mathrm{He}+{ }^{3} \mathrm{He} \rightarrow{ }^{4} \mathrm{He}+2 \mathrm{P}(12.9 \mathrm{MeV}) \\
& \mathrm{D}+{ }^{3} \mathrm{He} \rightarrow \mathrm{p}(14.68 \mathrm{MeV})+{ }^{4} \mathrm{He}(3.67 \mathrm{MeV}) \\
& \mathrm{P}+{ }^{11} \mathrm{~B} \rightarrow 3{ }^{4} \mathrm{He}+(8.7 \mathrm{MeV})
\end{aligned}
$$

Note: Aneutronic fusion is any form of fusion reaction where neutrons carry no more than $1 \%$ of the total released energy.

${ }^{3} \mathrm{He}$ fuel source is not abundant on earth, thus is only available via synthesis.

${ }^{3} \mathrm{He}$ is also obtainable from Lunar regolith mining or Jupiter atmosphere 10 PPB

abundance of ${ }^{3} \mathrm{He}$ fuel supply. 
The preferred fuel for space is the p-boron reaction: $\mathrm{p}+{ }^{11} \mathrm{~B} \rightarrow 3{ }^{4} \mathrm{He}+(8.7 \mathrm{MeV})$

${ }^{11}$ Boron is an abundant, inexpensive fuel stock and has the major advantage of a clean fuel for the primary fusion reaction, at the expense of reduced plant gain since currently $\mathrm{p}^{11} \mathrm{~B}$ energy gain estimates range from $\sim 5$ to 15 . Previously the parameters for harnessing aneutronic $\left(\mathrm{p}^{11} \mathrm{~B}\right)$ fusion had appeared significantly more exigent than for conventional (D-T) fusion fuel cycle. Yet recent scientific studies and engineering lab efforts applicable to micro-scale triggering of advanced fusion fuel solid targets has introduced new concepts with unique aspects that include demonstrations of attainable aneutronic [1] reactions. The successful application of aneutronic methodology to fusion propulsion plants for space deployment will ultimately offer the possibility of enhanced performance from nuclear gain as compared to existing chemical and even ion propulsive engines, while also providing a clean solution to planetary protection, Earth included considerations and requirements. Proton-triggered ${ }^{11}$ boron fuel $\left(\mathrm{p}^{-11} \mathrm{~B}\right)$ offers the potential for abundant ion kinetic energy for in-space vectored thrust applications as well as for direct energy conversion in specialized direct electrical energy conversion plants.

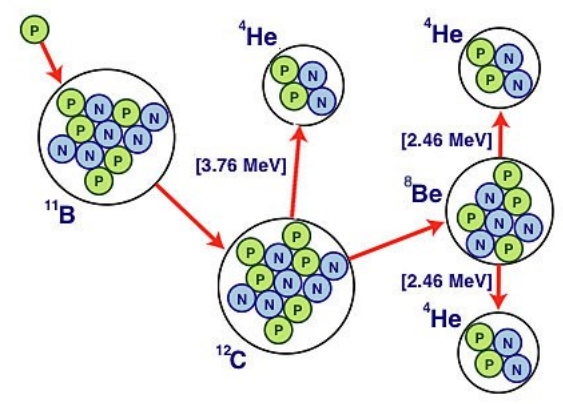

Figure 1. An energetic ( 163KeV) proton and a ${ }^{11}$ boron nucleus fuse kinematically to briefly form an excited ${ }^{12}$ carbon nucleus. The ${ }^{12}$ carbon immediately decays into one helium nucleus (alpha particle) and one ${ }^{8}$ Beryllium (excited state nucleus) which quickly decays into two additional 2.9 MeV alphas - each decay occurs within a few atto-seconds.

No neutrons are produced in this reaction, and the released energy is carried away by energetic doubly ionized alpha particles [2]. The energy yield, or nuclear gain potential, is characterized by specific reaction kinematics, temperature (ionic velocity), density and reactive species residence time, and collisional dynamics while in the plasma state. By using the methodology thus outlined, $\mathrm{p}-{ }^{11} \mathrm{~B}$ fuel can produce abundant ion kinetic energy [3] which can be utilized primarily for thrust and also for on-board direct power conversion for space craft. 


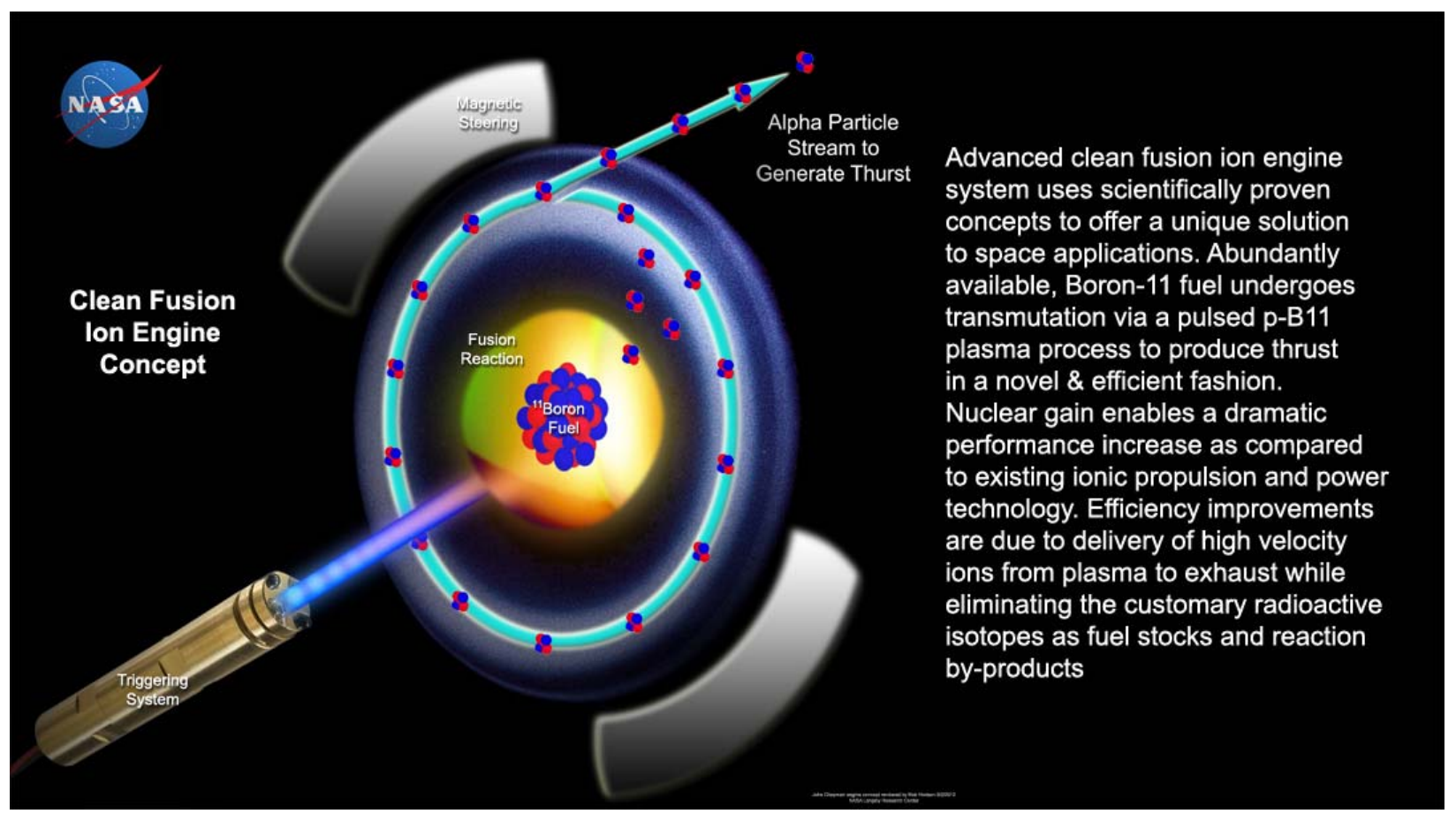

Figure 2. Thrust is realized via Lorentz reaction of electromagnetic forces coupled to the spacecraft frame

Lorentz equation : $\mathbf{F}=\mathrm{qE}+\mathrm{qv} \times \mathrm{B} \quad \mathrm{F}=$ force, $\mathrm{q}=$ charge, $\mathrm{E}=$ electric field, $\mathrm{B}=$ magnetic field

$\boldsymbol{F}=\boldsymbol{m} \boldsymbol{d} \boldsymbol{v} / \boldsymbol{d t}=\boldsymbol{q}(\boldsymbol{E}+\boldsymbol{v} \times \boldsymbol{B}), \boldsymbol{d} \boldsymbol{x} / \boldsymbol{d t}=\boldsymbol{v} \quad \mathrm{m}=$ mass, $\mathrm{v}=$ velocity, $\mathrm{x}=$ =position, $\mathrm{t}=$ time

The momentum of the energetic alpha particles provides clean, high ISP 900,000 as vectored thrust [4] such that $\mathrm{p}^{-11} \mathrm{~B}$ offers a clean fuel with well understood reaction kinematics. The $\mathrm{p}^{-11} \mathrm{~B}$ reaction is typically triggered via a pumped \& pinched plasma process with excited ions using hydrogen-boron fuel. Alternative means of triggering the $\mathrm{p}-{ }^{11} \mathrm{~B}$ reaction may provide a lower overall propulsion plant size and mass.

The Lawson triple product for the $\mathrm{p}^{-11} \mathrm{~B}$ reaction regarding plasma temperature-plasma density-confinement time can be met by Target Normal Sheath Acceleration (TNSA) of "trigger" ions using compact and lightweight hardware. The theoretical principles pertaining to this principle have been recently outlined as the Lawson-Woodward Theorem [5]. Triggering of the favored predictive yields from the $\mathrm{p}-{ }^{11} \mathrm{~B}$ reaction is optimized at two resonant energy levels corresponding to $\sim 163 \mathrm{keV}$ and $\sim 560 \mathrm{KeV}$ (p${ }^{11} \mathrm{~B}$ center of mass). 
These ionic energy levels are readily attainable via high contrast ratio pulsed laser triggering (TNSA) of selectively layered targets. Photon impact excitation of ionic species $(>1 \mathrm{MeV})$ is achieved in the target plane resulting from picosecond duration, high contrast ratio Chirped Pulse Amplified (CPA) laser abrupt wavefront target-normal impingement. The photonically-induced ion acceleration process which occurs at the target material focal point has been demonstrated to spawn fusion reactions in the adjacent composite.

The induced "hot" electrons are expelled from the target due to the intense electric field $\sim$ TeraVolt per meter. This induces a coulombic self-repulsive effect or instantaneous ionic explosion at the focal point. The TNSA ions create plasma ejecta which erupts from the (thin) electron-depleted substrate, see Figure 3.

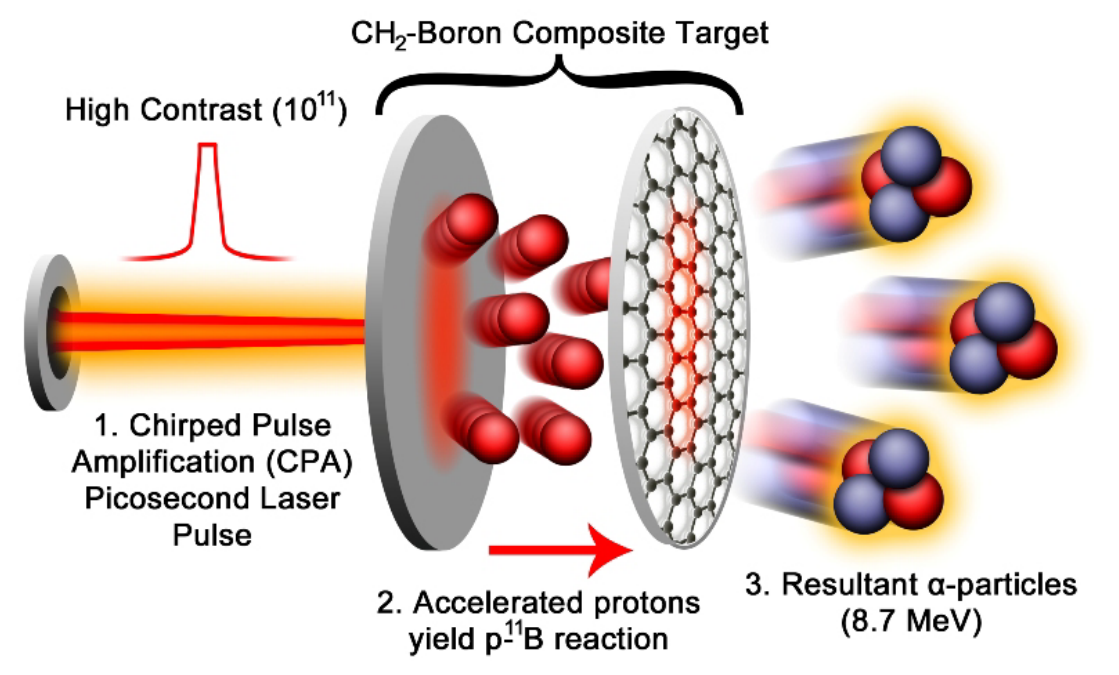

Figure 3. Composite target employing ${ }^{11}$ Boron layer impregnation in conjunction with triggering of the reaction by custom CPA laser to achieve Target Normal Sheath Acceleration (TNSA) of precursor ions. This induces a laser triggered plasma-jet from a solid target in vacuum.

Effective fusion cell design must be optimized to maximize the vector velocity of energetic ionic ejecta destined to exit via the exhaust nozzle. A collimated ionic plume of energetic reactant alpha particles would thus constitute the physical basis of a finite thrust impulse.

\section{Energy considerations}

Power efficiency: $\eta_{e}=P_{e} / P_{\text {in }}$

Pin is the total (laser power) input (watts)

$\mathbf{P e}_{\mathrm{e}}$ is the power in exhaust stream of ejected $3 \mathrm{MeV}$ alphas

[7] Power efficiency entails the aspects of reaction triggering and associated power loss burdens such as energy spent in lasing medium excitation, ionization and target level internal losses. The quantum efficiency of (Fig.3) CPA laser systems capable of producing high contrast ratio picosecond pulses is below $1 \%$, however for a rocket thrust application (as contrasted with a power 
production plant) the $\eta_{e}$ ratio can be less than $100 \%$. The incremental thrust from a laser triggered $\mathrm{p}-{ }^{11} \mathrm{~B}$ target, assuming $\sim 10^{5}$ Alphas from a single laser pulse has been estimated to yield a few pico-Newton impulse per laser pulse from a 10 micron square target area. High pulse rate laser systems coupled with multiple square centimeters of active target area could effectively augment the effective thrust level towards Newton magnitude levels, particularly in conjunction with increased alpha yields from optimized target designs. Recent advances in laser technology indicate possibility of higher laser quantum efficiencies (>25\%) and higher femtosecond pulse train rates ( 75MhZ) .

Bremstrahlung radiation and non-productive plasma also result in losses as well as particle collisions with the structure represent additional power losses from the propellant exhaust stream. Power is also lost in transverse momentum resulting in exhaust stream spreading.

Assume the mass efficiency to be unity since ideally all the propellant "ash" leaves the engine.

$\alpha=\mathrm{M} / \mathrm{W}$ (kWthrust / kgreactor)

$\alpha=$ Power plant specific mass expressed in units of kilograms per watt

Future development and the availability of high efficiency short pulse laser systems may result in overall gains in $\alpha$ and ISP values that may make the $\boldsymbol{A}$-LIFT (Aneutronic Laser Induced Fusion Thruster) offering ISP 900,000 approach an attractive alternative to previous fusion $\sim 1-10 \mathrm{~kW} / \mathrm{kg}$ or ionic (ISP range from 2000 - 100,000 propulsion for InSpace thrust applications.

Table 1. Fusion Reactor Designs for Space Applications ${ }^{[10]}$

\begin{tabular}{|c|c|c|c|}
\hline First Author & Year & Configuration & $\begin{array}{c}\text { Specific Power } \\
\text { (kW/kg) }\end{array}$ \\
\hline Borowski & 1987 & Spheromak & 10.5 \\
\hline Santarius & 1988 & Tandem Mirror & 1.2 \\
\hline Chapman & 1989 & FRC & -- \\
\hline Haloulakis & 1989 & Colliding Spheromaks & -- \\
\hline Bussard & 1990 & Riggatron Tokamak & 3.9 \\
\hline Bussard & 1990 & Inertial-Electrostatic & $>10$ \\
\hline
\end{tabular}




\begin{tabular}{|c|c|c|c|}
\hline Teller & 1991 & Dipole & 1.0 \\
\hline Carpenter & 1992 & Tandem Mirror & 4.3 \\
\hline Nakashima & 1994 & FRC & 1.0 \\
\hline Kammash & 1995 & Gas Dynamic Trap & $21(\mathrm{D}-\mathrm{T})$ \\
\hline Kammash & 1995 & Gas Dynamic Trap & $6.4\left(\mathrm{D}-{ }^{3} \mathrm{He}\right)$ \\
\hline
\end{tabular}

Conceptual designs of magnetic fusion reactors for space propulsion from past decades have generally calculated specific powers of 1--10 $\mathrm{kW}_{\text {thrust }} / \mathrm{kg}_{\text {reactor. }}$ The projected specific powers for selected designs appear in the table. Note: Widely varying assumptions and levels of optimism have gone into these conceptual designs and the resulting specific powers.

\section{Direct conversion of Energy:}

An important related aspect making aneutronic fusion reactions amenable to space missions is that most of the energy is released in the form of charged particle kinetic energy. This kinetic energy can be converted directly into electricity via conversion techniques based on mature technology derived from other fields, such as microwave technology, resonance-tuned rectennae [8] which entails equipment that is more compact and potentially cheaper than that involved in conventional thermal production of electricity. Various fuels D-T, D- ${ }^{3} \mathrm{He}, \mathrm{p}^{-}{ }^{11} \mathrm{~B}$ and ${ }^{3} \mathrm{He}-{ }^{3} \mathrm{He}$ have also been investigated and compared [9] for favorable energy yields in the IEC reactor.

\section{Potential Impact}

Ultimately advances in clean fusion plant technology using aneutronic fuels offer the potential of far reaching impact upon next generation space missions. Such new technologies could be utilized in satellite station keeping, in the exploration of planets and asteroids and in terrestrial energy applications. Energetic alpha particle "exhaust" momentum can be used directly to produce high ISP thrust and also offers the possibility of direct power conversion into electricity. $\mathrm{p}^{-}{ }^{11} \mathrm{~B}$ is an advanced fusion plant fuel with well understood reaction kinematics but still requires implementation of new conceptual thinking for optimal implementation. Development of aneutronic fusion plants should also be persued [6] to secure potentially more energetic and abundant sources of clean, sustainable, high density energy, with applications for powering extended space exploration missions. Over recent decades the methodology for effective harnessing of propulsive energy via fusion has made steady progress. But scientific studies and engineering efforts in advanced fusion systems design and development have lately introduced new concepts (i.e. pulsed laser triggering) each with unique aspects and characteristics which offer new oportunity for clean in-space propulsion and power generation. 
PIC (Particle in Cell) simulations at the unit cell level offers the possibility for optimization of reaction unit cell characteristics to predict customized plant configurations which may ultimately lead to dramatic fusion plant size and mass reductions along with potential cost reductions. Reductions in expenses associated with conversion of fusion energy directly to thrust as well as electrical power production in fusion plants for space applications planetary or terrestrial is desireable.

Aneutronic fusion processes via readily available boron fuel will also best satisfy Planetary Protection requirements for minimization of potential contamination of either surface or planetary magnetosphere due to radioactive emissions or accidentally incurred leaks.

\section{Relevance to NASA}

Future vision for Aneutronic Fuels entails applications of Advanced Fusion Fuel Propulsion aligns conceptually with NASA's In-Space Propulsion Roadmap. Major state of the art improvements in NASA mission range, endurance and reliability will be manifested when physical limitations associated with chemical and fission plants are overcome. Successful exploitation of fusion technology will have direct relevance to a broad class of NASA exploration missions as well as upon the commercial space enterprise. Successful application of aneutronic fusion would greatly reduce problems associated with neutron radiation such as ionizing damage, neutron activation, and requirements for biological shielding, remote handling, and safety.

Neither the fuel nor the reaction products from aneutronic fusion processes are radioactive.

\section{References \& Citations}

[1] Fusion energy without radioactivity: laser ignition of solid hydrogen-boron (11) fuel Heinrich Hora, George H. Miley, M. Ghoranneviss, B. Malekynia, N. Azizic and Xian-Tu. Hed (March 2010)

[2] Belyaev, V.S., V.I. Vinogradov, A.P. Matafonov, S.M. Rybakov, V.P. Krainov, V.S. Lisitsa, V.P. Andrianov, G.N. Ignatiev, V.S. Bushuev, A.I. Gromov, A.S. Rusetsky, V.A. Dravin "Excitation of Promising Nuclear Fusion Reactions in Picosecond Laser Plasmas" Physics of Atomic Nuclei 72, no. 7 (2009): 10771098.

[3] Hora, H. Miley, G.H. "Hydrodynamic Studies of Laser Fusion using Plasma Block Ignition Driven by Nonlinear Ponderomotive Forces IOP" (2010), (accessed April 19, 2011).

[4] Gilland, James H.. "Mission and System Optimization of Nuclear Electric PropulsionUMich". 91-038. (1991)

[5] Nakajima, Kazuhisa. "Acceleration and Focusing by superstrong laser fields and their applications2nd ORION WORKSHOP SLAC". (2003),

[6] Thomas, Robert, Yang Yang, G.H. Miley, F.B. Mead "Advancements in Dense Plasma Focus (DPF) for Space Propulsion." AIP Conf. Proc. 746, no. (2005): 536-543. 
[7] Miller, David B. , “Plasma thrusters for space propulsion”, IEEE Spectrum, JUNE (1965)

[8] Perkins, Miley, Logan, “Novel Fusion Energy Conversion Methods” Nuc. Inst. Methods Phys. Res. A271 (1988)

[9] Kulcinski, G.L. http://fti.neep.wisc.edu/neep533/SPRING2004/lecture26.pdf

[10] EP602 Course Notes (Fall 1997) Resources from Space Lecture \#32: Opening the Solar-System Frontier," Fusion Propulsion" Santarius and Logan, 1998. 